\title{
Path Planning for Mobile Robots Based on Hybrid Architecture Platform
}

\author{
Ting Zhou, Xiaoping Fan \& Shengyue Yang \\ Laboratory of Networked Systems, Central South University, Changsha 410075, China
}

Zhihua Qu

Laboratory of Networked Systems, Central South University, Changsha 410075, China

$\&$

Department of Electrical and Computer Engineering, University of Central Florida

Orlando, FL 32816, USA

\begin{abstract}
In this paper, a hybrid architecture is used to develop the software platform of path planning of mobile robots. It consists of four levels: the decision-making layer, the behavior layer, the command parsing layer and the hardware communication layer. The whole framework of the decision-making system is based on the global planning, which is realized by using the ant colony algorithm. In the process of movement, the robot detects the real-time local information using the sensors mounted on it, and calls diffident behaviour objects to revise the global path for diffident situations. The experimental results show that the mobile robot has good capacity for unexpected situations in the process of moving along the optimal path.
\end{abstract}

Keywords: Robot, Hybrid architecture, Ant colony algorithm, Path planning

\section{Introduction}

The systematic structure of control software adopted by the mobile robots is mainly divided to such different kinds: programmable architecture, subsumption architecture and hybrid architecture. The programmable architecture performs in passing series according to a sequence of actions presented by the planning tool given the original and objective status. It adapts to the environment which has the characteristics of having enough environmental information, the accurate information without much dynamics, the environmental diversification with non-symbolization, however, which can not adapt to the complex environment and deal with the unknown and unexpected situations. The subsumption architecture, which also namely responding models, means that the complex assignment is decomposed to a series of relatively simple and special actions, which all control one aspect of integrated target based on the information of sensors. The robot system based on behaviors can make quick responses according to the ambience diversification, which has good real time property, but it does not make global plan for the assignment, which so can not assure the realization of objects is optional. The hybrid architecture is the integrated system of plans and actions, which not only is sensitive to the environment but also assure to the realization efficiency of objectives. Usually, the hybrid architecture has two kinds of models: one kind is that the whole framework of the decision-making system is based on the planning and it is then governed by behavioral models in the dynamic situation; the other one is that the whole framework of the decision-making system is based on behaviors, which applies planning models in some special behaviors. To sum up, the desirable goal of hybrid architecture is that it integrates the advantages of programmable architecture and subsumption architecture and avoids the disadvantages. This paper adopts the first model of hybrid architecture to construct the path planning platform of robots. The ant colony algorithm is applied for the global path planning. All kinds of behavioral models are used for processing in the dynamic situations to ensure that the robots can arrive at the targets safely and quickly.

\section{The dynamic path planning of robots}

Zhu, Baoqing et al. (2005) demonstrated detailedly about the timeliness, accessibility and convergence of the ant colony algorithm applied in the global path planning of robots, which is not referred repeatedly. Many scholars have discussed the path planning of robots in the unknown situations(Fan, Xiaoping, Li, Shuangyan \& Chen, 2005)(Lei, Lin, Wang, Houjun \& Wu, Qinsong, 2006)(Hong Song, Lianjun Hu, Xiaohui Zeng. 2009)(Zhuang, Huizhong. 2006). This paper plans one collision-free path making using of the ant colony algorithm during the dynamic planning, according to which the robots move. In the process of movement, the sensors are used to detect the information of dynamic obstacles in real time. For the simple situation, we suppose only one dynamic 
obstacle exist and it moves uniformly.

The dynamic planning algorithm is as follows:

Step 1: the robots moves forward one step. If the current grid is the objective one, then switch to step 7. Otherwise, switch to step 2.

Step 2: The sensor once detects the obstacles when it moves forward one step and it distinguishes the static and the dynamic obstacles. If they are the static obstacles, then switch to step 1. Otherwise, if they are the dynamic ones, the information $p_{t}(x, y)$ of location where the obstacles arise is recorded and then switch to step 3 .

Step 3: This step judges whether the robot locates in the safe area. If the distance between the obstacles and the robot is less than $0.3 \mathrm{~m}$ or the infrared sensor detects the obstacles, then switch to step 4 for the reason of safety. If it locates in the safe area, then look over whether the information record $p_{t-1}(x, y)$ about the obstacle exists whether or not and continue to detect after $0.2 \mathrm{~s}$ to obtain the location $p_{t}^{\prime}(x, y)$ of obstacles. Switch to step 5. If no obstacles are detected, it is believed that the obstacles have deviated from the detectable range of robot or the ultrasonic sensors can not detect the obstacles, then switch to step 1 .

Step 4: The robot searches the grid according to the direction (clockwise or anticlockwise) which most closes to the direction of end-point. The grid which does not have obstacle at first is just the moveable grid. Move this grid to switch to step 6 .

Step 5: Making use of the known information of obstacles to predict collision points. If the location of next moment is in the planning path, then switch to step 6. If it does not have point of intersection with the planning path, then switch to step 1.

Step 6: Beginning with the current grid to plan the path again. If the current grid is the objective one, then switch to step 7, otherwise switch to step 1 .

Step 7: The robot arrives at the objective grid and stops moving.

\section{The hybrid architecture platform}

The software developing platform applies the hybrid architecture based on the planning. It mainly divided into four layers: the decision-making layer, the behavior layer, the command parsing layer and the hardware communication layer. It is shown as figure 1.

\section{The decision-making layer}

The decision-making layer is the highest local layer of robots. The decision-making objects organizing all kinds of behaviors through the local sensor data, the running state of robots and the instruction data sent from the remote end. In this paper, the whole framework of the decision-making layer is based on the global path planning, which is realized by using the improved ant colony algorithm. The environment modeling adapts the grid method.

During the process of realizing the assignment of path planning of robot, the decision-making object acquires the beginning and ending data from the input interface and it makes use of the ant colony algorithm to plan the optional path according to the input environmental information, which is transmitted to cruising behavioral objects and connected with the objects of protocol layer. The cruising behavioral objects drive the robot to run according to the planned path till the objective point. In the process of moving, the cruising behavioral objects will hand the control rights to the colliding points predicting behaviors or the urgent avoiding obstacle behaviors provisionally in the situation of obstacles blocking off. After avoiding the obstacles, it is decided that the control rights are handed to the cruising behavior to continue moving or the decision-making objects plan the path again according to different situations.

\section{The behavior layer}

The behavior layer is the objects of some abstract behaviors. These objects plan the local behaviors aiming at the sensor data of consultative objects and manipulate the objects to send control instructions to robots. The behavior layer includes the colliding point predicting behavior, the urgent avoiding obstacle behavior, the cruising behavior and so on.

\section{The command parsing layer}

The command parsing layer is the core of control architecture of robots. The targets of command agreement compiles the control command of robots substantiality and analytically transforms the sensor data and the state data of robot returned from the lower computer. 


\section{The hardware communication layer}

The hardware communication layer is the targets which communicate with the hardware equipment directly.

\section{The experimental result and analysis}

Some static obstacles are located in the experimental room and the environmental information is transformed to the maps, in which the information about static obstacles and the location and end point of robots are regarded as the known information as shown in figure 3 and 4 . The robot is located at $(3,0)$ and the end point is $(7,11)$ and the step of robot is $0.6 \mathrm{~m}$. According to the above information, the off-line whole path planning is made out. The planned path is shown as figure 2(a).

The robot moves according to the planned path. It moves from the beginning point to detect the obstacle information in real-time. When it moves to the location of $(4,3)$, the third sensor detects one dynamic obstacle in the front of $1.317 \mathrm{~m}$, which is also $(4,6)$, then continues detecting in $0.2 \mathrm{~s}$. The location of obstacle does not change much, so it moves according to the planned path. When it moves to the point of $(5,4)$, it does not detect the dynamic obstacle because of the angle of detectors. When the robot moves to $(5,5)$, the $24^{\text {th }}$ detector detects the obstacle in the front of $0.402 \mathrm{~m}$ and through calculating, it is believed that they may collide at the point of ( 6 , 6). So from the beginning point $(5,5)$ to end at the point of $(7,11)$ it has to plan the path again. The planned path is shown as figure 2(b). The actual path is shown as figure 3(a)-(e), where the robot moving according to the $Y$ direction is the experimental one and the robot moving according to the $\mathrm{X}$ direction is the dynamic obstacle.

In many experimental processes, the robot can move according to the planned path. If it meet the dynamic obstacle, the decision-making layer can judge different situations to call for different behavioral objects through the judgment results. This paper also designs the reflection of robot when the dynamic obstacles suddenly speed up and move towards it. The experimental results show that the robot can enter into the urgent avoiding obstacle program to avoid the obstacles temporarily

\section{Conclusions}

This paper applies the hybrid architecture to construct the path planning platform of robot, in which the path planning algorithm is designed. The whole framework of the decision-making system is based on the whole planning, which is realized using the ant colony algorithm. During the process of movement, the real-time local information is detected through the sensors carried on the robot. The whole path is amended aiming at calling for different behavioral objects in different situations. The experimental results show that the mobile robot has good capacity for unexpected situations in the process of moving along the optimal path.

Usually, the scene considered in the local path planning algorithm is relatively simple and only one dynamic obstacle moving uniformly is considered. When there are many dynamic obstacles, the calculation is increasing and the problem of path planning has to be studied and tested further.

\section{References}

Zhu, Baoqing. (2005). Ants predictive algorithm for path planning of robot in a complex dynamic environment. Chinese Journal of Computers, 2005, 28(11): 1898-1906.

Fan, Xiaoping, Li, Shuangyan \& Chen, Tefang. (2005). Dynamic obstacle-avoiding path plan for robots based on a new artificial potential field function. Control Theory \& Applications, 2005, 22(5): 703-707.

Lei, Lin, Wang, Houjun \& Wu, Qinsong. (2006). Improved Genetic Algorithms Based Path Planning of Mobile Robot under Dynamic Unknown Environment. In: Proceedings of the 2006 IEEE International Conference on Mechatronics and Automation, Luoyang, China, June 25-28, 2006: 1728-1732.

Hong Song, Lianjun Hu, Xiaohui Zeng. (2009). Research on Path Planning for the Mobile Intelligent Robot [C]. In: 2009 World Congress on Computer Science and Information Engineering, 2009: 121-124.

Zhuang, Huizhong. (2006). On-line real-time path planning of mobile robots in dynamic uncertain environment. Hangzhou: Zhejiang University, 2006: 36- 41. 


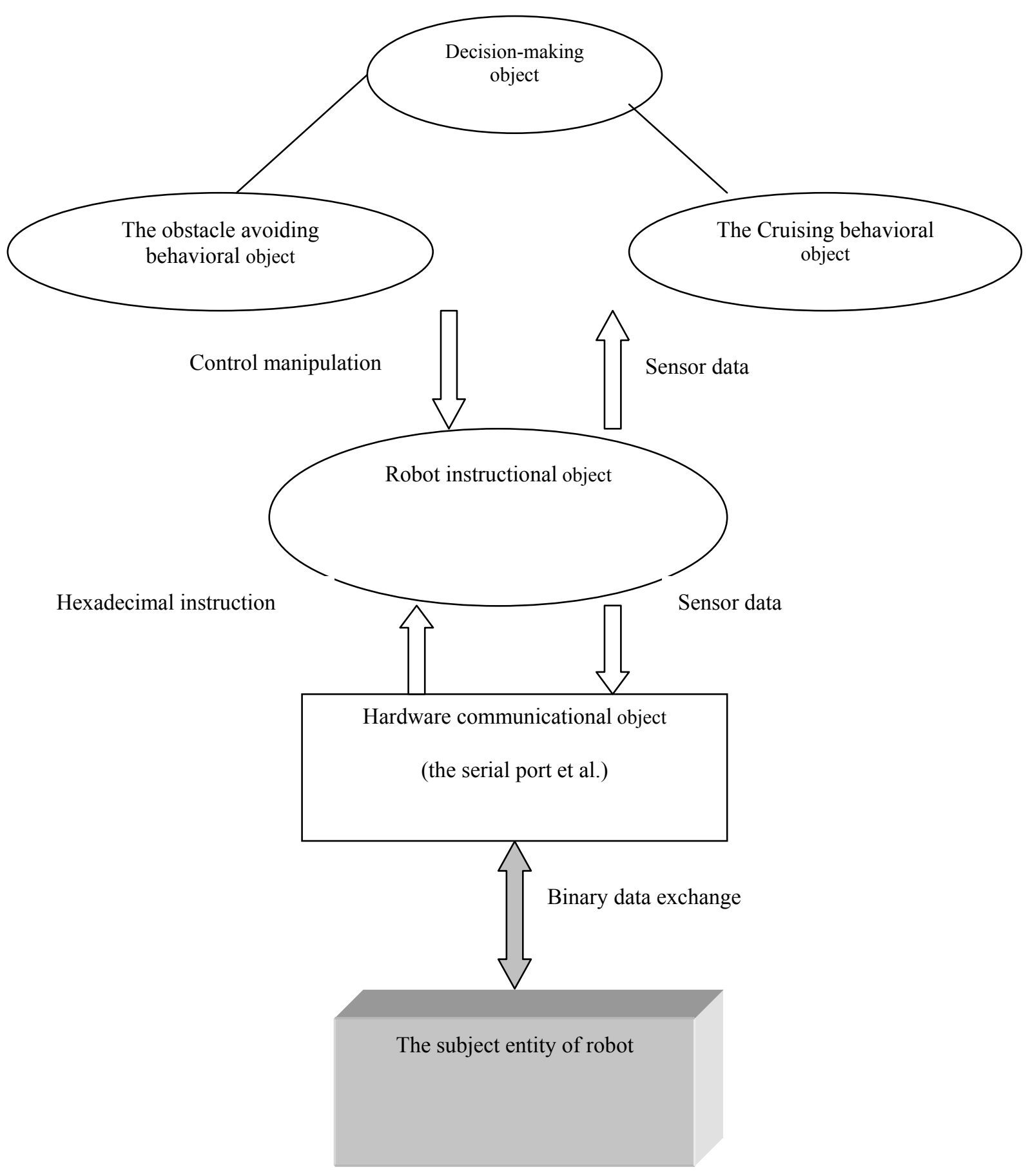

Figure 1. The experimental platform of robots 


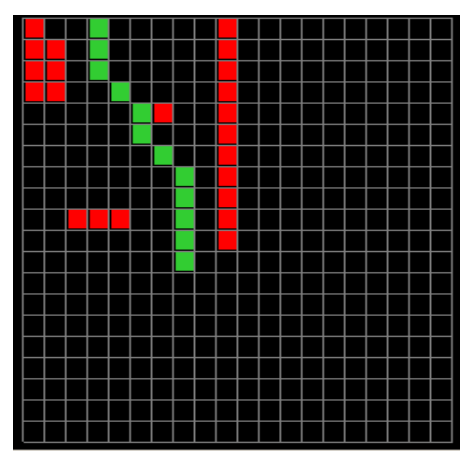

(a) the original path

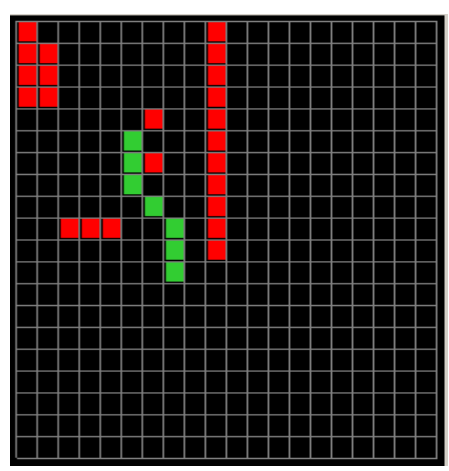

(b) the new path after re-planning

Figure 2. the path planning results

Note: the red grids denote the obstacles and the green ones denote the newly planning path and the additional red obstacles denote the predictive colliding points.

dynamic obstacles static obstacles mobile robot

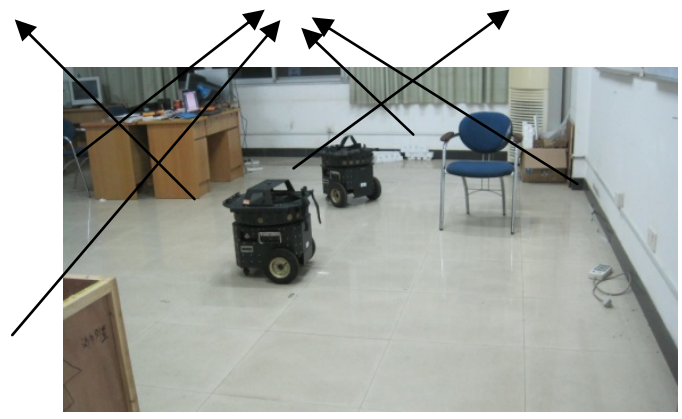

(a)

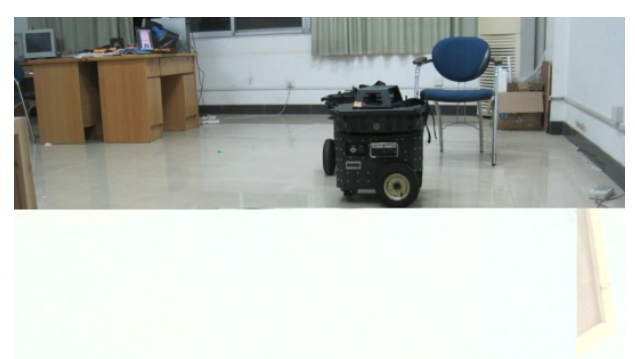

(c)

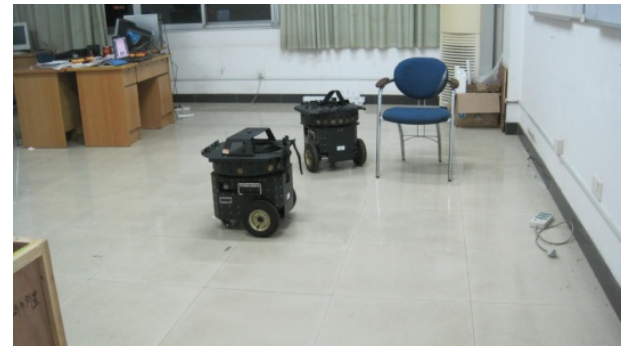

(b)

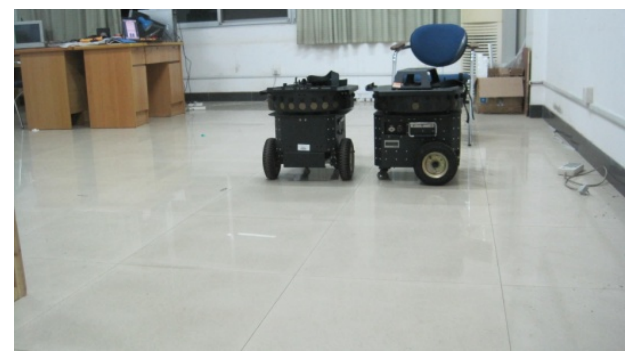

(d)

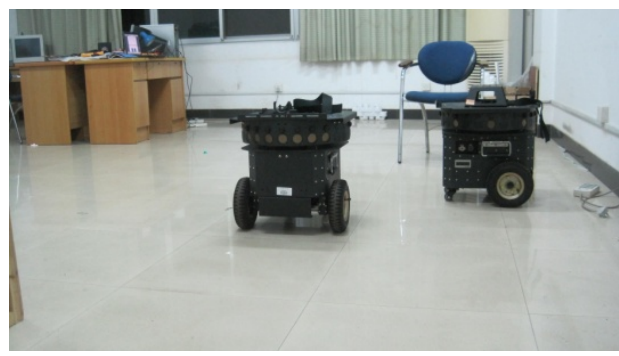

(e)

Figure 3. The experimental instances of robot in the process of actual movement 